\title{
Blocking VEGF/Caveolin-1 signaling contributes to renal protection of fasudil in streptozotocin-induced diabetic rats
}

\author{
Jing $\mathrm{JIN}^{1, \text { \# }}$, Chao PENG ${ }^{1, \text { \# }}$, Su-zhen WU ${ }^{1}$, Hong-min $\mathrm{CHEN}^{1}$, Bai-fang ZHANG ${ }^{1,2, *}$ \\ ${ }^{1}$ Department of Biochemistry, Wuhan University School of Basic Medical Sciences, Wuhan 430071, China; ${ }^{2}$ Hubei Provincial Key \\ Laboratory of Developmentally Originated Disease, Wuhan 430071, China
}

\begin{abstract}
Aim: RhoA/ROCK signaling plays an important role in diabetic nephropathy, and ROCK inhibitor fasudil exerts nephroprotection in experimental diabetic nephropathy. In this study we investigated the molecular mechanisms underlying the protective actions of fasudil in a rat model of diabetic nephropathy.

Methods: Streptozotocin (STZ)-induced diabetic rats, to which fasudil or a positive control drug enalapril were orally administered for 8 months. Metabolic parameters and blood pressure were assessed during the treatments. After the rats were euthanized, kidney samples were collected for histological and molecular biological studies. VEGF, VEGFR1, VEGFR2 and fibronectin expression, and Src and caveolin-1 phosphorylation in the kidneys were assessed using RT-PCR, Western blot and immunohistochemistry assays. The association between VEGFR2 and caveolin-1 was analyzed with immunoprecipitation.

Results: Chronic administration of fasudil (30 and $100 \mathrm{mg} \cdot \mathrm{kg}^{-1} \cdot \mathrm{d}^{-1}$ ) or enalapril $(10 \mathrm{mg} / \mathrm{kg}$, bid) significantly attenuated the glomerular sclerosis and albuminuria in the diabetic rats. Furthermore, fasudil treatment prevented the upregulation of VEGF, VEGFR1, VEGFR2 and fibronectin, and the increased association between VEGFR2 and caveolin-1 in the renal cortices, and partially blocked Src activation and caveolin-1 phosphorylation on tyrosine 14 in the kidneys, whereas enalapril treatment had no effects on the VEGFR2/ Src/caveolin-1 signaling pathway.

Conclusion: Fasudil exerts protective actions in STZ-induced diabetic nephropathy by blocking the VEGFR2/Src/caveolin-1 signaling pathway and fibronectin upregulation. Thus, VEGFR2 may be a potential therapeutic target for the treatment of diabetic nephropathy.
\end{abstract}

Keywords: diabetic nephropathy; glomerular sclerosis; RhoA/ROCK signaling; fasudil; enalapril; VEGF; Src; caveolin-1

Acta Pharmacologica Sinica (2015) 36: 831-840; doi: 10.1038/aps.2015.23; published online 4 May 2015

\section{Introduction}

Diabetic nephropathy is one of the most serious microvascular complications of diabetes mellitus. The current treatments for diabetic nephropathy include optimization of glucose and blood pressure control by targeting the renin-angiotensin system (RAS) with angiotensin-converting enzyme (ACE) inhibitors and/or angiotensin II receptor blockers. Despite these measures, renal damage progresses in many patients. Novel strategies are therefore required, and some recent studies suggest that the inhibition of small G-protein RhoA and its downstream effector, Rho-associated kinase (ROCK), is a promising approach $^{[1-5]}$.

RhoA is the best studied member of the Rho GTPase family.

\footnotetext{
\# These authors contributed equally to this work.

* To whom correspondence should be addressed.

E-mail zbfwq@whu.edu.cn

Received 2014-11-14 Accepted 2015-03-10
}

RhoA behaves as a "molecular switch" that cycles between an inactive GDP-bound state and an active GTP-bound state ${ }^{[6]}$. ROCK is a major RhoA effector. RhoA/ROCK signaling regulates numerous cell functions, including vascular contraction, actin cytoskeleton reorganization, cellular morphology, motility, adhesion and proliferation ${ }^{[7,8]}$. Recent studies have indicated that RhoA/ROCK signaling is an important contributor to diabetic nephropathy ${ }^{[9,10]}$. Fasudil, a relatively specific ROCK inhibitor, has nephroprotective actions during experimental diabetic nephropathy ${ }^{[11-13]}$. The possible mechanisms include the attenuation of diabetes-induced increases in renal TGF- $\beta$, CTGF and extracellular matrix (ECM) protein expression resulting in slower glomerulosclerosis and interstitial fibrosis development. Hemodynamic mechanisms and antiproteinuric effects might also be involved. Fasudil also inhibits other kinases, such as protein kinase C isoforms ${ }^{[14]}$, which might contribute to the nephroprotective actions. However, the mechanisms underlying the activation of RhoA/ROCK 
signaling during diabetic nephropathy remain unknown, and the molecular mechanisms of fasudil should be characterized in more detail.

The cytokine vascular endothelial growth factor (VEGF) is an important regulator of normal kidney functions ${ }^{[15]}$. Recently, VEGF was demonstrated to be upregulated by high glucose in mesangial cells and was implicated in the pathogenesis of diabetic nephropathy. Increased VEGF involves glomerular hypertrophy, proteinuria and ECM production $^{[16-18]}$. Peng et al demonstrated that the upregulation of VEGF transcription in mesangial cells induced by glucose was prevented by the Rho inhibitor Y-27632 indicating that VEGF upregulation is possibly dependent on RhoA/ROCK signaling $^{[10]}$. There are three known VEGF receptors: VEGFR1/fmsrelated tyrosine kinase 1 (Flt1), VEGFR2/kinase insert domain receptor (KDR), and Neuropilin-1 ${ }^{[19]}$. VEGF elicits its biological functions by activating specific transmembrane receptors (VEGFR1 and VEGFR2) with intrinsic tyrosine kinase activity $^{[20]}$. Neuropilin-1 has no tyrosine kinase activity. There is only a small amount of data concerning the role of VEGF signaling pathways during diabetic nephropathy. The present study focused on the potential mechanisms of fasudil inhibition of VEGF upregulation and its receptors (VEGFR1 and VEGFR2) in the renal cortex of streptozotocin (STZ)-induced diabetic rats. The effects of fasudil were compared to the ACE inhibitor enalapril, which is an established therapeutic agent for both clinical and experimental diabetic nephropathy.

\section{Materials and methods}

\section{Diabetic rat model}

Experiments were performed with male Sprague-Dawley rats weighing 200-225 g (Animal Center, Wuhan University, China) in accordance with the official recommendations of the Chinese Community Guidelines. Diabetes was induced with a $55 \mathrm{mg} / \mathrm{kg}$ STZ (Sigma, St Louis, Missouri, USA) injection by tail vein. Control rats $(n=10)$ were injected with equal volume of citrate buffer. Hyperglycemia (blood glucose $>20 \mathrm{mmol} / \mathrm{L}$ ) was confirmed ( $2 \mathrm{~d}$ after injection) with a reflectance meter (One Touch, Lifescan, Milpitas, California, USA). Diabetic rats were randomly divided into five groups: untreated, lowdose fasudil (10 $\mathrm{mg} \cdot \mathrm{kg}^{-1} \cdot \mathrm{d}^{-1}$; Sigma), middle-dose fasudil (30 $\left.\mathrm{mg} \cdot \mathrm{kg}^{-1} \cdot \mathrm{d}^{-1}\right)$, high-dose fasudil $\left(100 \mathrm{mg} \cdot \mathrm{kg}^{-1} \cdot \mathrm{d}^{-1}\right)$, and enalapril (10 mg/kg twice daily; Merck, Darmstadt, Germany) by intragastric gavage (ig). Treatment continued for 8 months. All rats had free access to regular lab chow and water. The diabetic rats also received low dose insulin if required to prevent ketonuria as assessed with a dipstick (Bayer Multistix) but to maintain hyperglycemia $>20 \mathrm{mmol} / \mathrm{L}$. The blood glucose levels were monitored weekly in all diabetic rats. Urine samples were collected for $24 \mathrm{~h}$ in metabolic cages at month 1, 3, and 8. Systolic blood pressure was determined by the tail-cuff method monthly. After anesthesia induction with an injection with sodium pentobarbital $(50 \mathrm{mg} / \mathrm{kg}$, ip), blood was collected from the aorta, and the kidneys were removed. The kidney samples were rapidly excised, weighed, frozen in liquid nitrogen and stored at $-80^{\circ} \mathrm{C}$. Renal hypertrophy was assessed by the kidney to body weight ratio $(\mathrm{mg} / \mathrm{g})$ at the time of euthanasia. Some samples were fixed for immunohistochemistry or transmission electron microscopy.

\section{Biochemical analysis and morphological studies}

Blood glucose, serum or urinary creatinine, and urinary albumin levels were determined by the Department of Clinical Laboratory at Zhongnan Hospital, Wuhan University. Ccr $\left(\mathrm{mL} \cdot \mathrm{min}^{-1} \cdot 100 \mathrm{~g}\right.$ body weight $\left.{ }^{-1}\right)=$ urine creatinine $\times$ urine volume $\left(\mathrm{mL} \cdot \mathrm{min}^{-1}\right) /$ serum creatinine per $100 \mathrm{~g}$ body weight. For morphological studies, the excised kidneys were fixed in $10 \%$ formalin, dehydrated through a graded series of ethanol, embedded in paraffin, cut into $4 \mu \mathrm{m}$ thick slices and mounted on glass slides. Some sections were stained with hematoxylin and eosin (H\&E) to observe the tissue morphology. Some fixed sections were stained with periodic acid-Schiff (PAS) reagent. Briefly, the deparaffinized sections were oxidized in $0.5 \%$ periodic acid solution for $5 \mathrm{~min}$ and stained in Schiff reagent for $15 \mathrm{~min}$. After washing in lukewarm tap water for $5 \mathrm{~min}$, the sections were dehydrated through a graded series of ethanol. Finally, the cover slips were placed on the glass slides with a mounting medium (neutral balsam), and the slides were observed under a light microscope.

\section{Glomerulosclerosis score}

In total, 50 full-sized glomeruli were chosen randomly from 2-3 slides (PAS-stained sections) and were assessed at 400x magnification. The glomerular sclerosis was scored by an experimenter that was blind to the treatment groups) as follows: $0=$ no sclerosis, $1=25 \%$ glomerular area involved, $2=50 \%$, $3=75 \%, 4=100 \%{ }^{[21]}$. The sections were scored by visual inspection. A score was assigned for each glomerulus, and these values were averaged to obtain a final score for each group.

\section{Immunohistochemistry}

Formalin-fixed, paraffin-embedded renal cortical sections (4 $\mu \mathrm{m})$ were routinely deparaffinized and rehydrated in distilled water. First, the deparaffinized sections were subjected to heat-induced antigen retrieval by incubation in citrate buffer solution ( $\mathrm{pH} \mathrm{6}$ ) for $20 \mathrm{~min}$ at $95^{\circ} \mathrm{C}$, cooled to room temperature, and washed with PBS. These sections were then treated with $3 \% \mathrm{H}_{2} \mathrm{O}_{2}$ for 10 min to block endogenous peroxidase activity. After rinsing, the sections were blocked with 3\% BSA in PBS and were incubated with monoclonal anti-rat VEGFR1 (1:100, Santa Cruz Biotechnology, Dallas, Texas, USA) or monoclonal anti-rat VEGFR2 (1:100, Santa Cruz Biotechnology) at $4^{\circ} \mathrm{C}$ overnight. Thereafter, the sections were incubated within secondary antibody for $30 \mathrm{~min}$. After washing with PBS, the sections were stained with a 3,3'-diaminobenzidine solution $(\mathrm{DAB})$. Counterstaining for the nucleus was performed with hematoxylin. A negative control was performed with the absence of primary antibody. Positive staining (dark brown) was quantified by two investigators in a blinded manner under high-power magnification $(\times 400)$ using the image analysis software Image-Pro Plus 7.0. The data are expressed as the average intensity of the threshold area. 


\section{Transmission electron microscopy}

A small piece of kidney cortex was harvested for transmission electron microscopy (TEM). The tissue was fixed with $2.5 \%$ glutaraldehyde, post-fixed with $1 \%$ osmium tetroxide, and stained en bloc with $2 \%$ aqueous uranyl acetate. The samples were then processed by an EM facility at Wuhan University and viewed with an HT7700 transmission electron microscope (Hitachi, Tokyo, Japan) at $40-80 \mathrm{kV}$. The basement membrane thickness was assessed on the peripheral loops, which were photographed randomly at $10000 \times$ magnification. The harmonic mean of measurements at $80-100$ points crossing a grid from 1-2 glomeruli was calculated.

\section{Protein extraction and Western blot}

Kidney cortices or glomeruli were homogenized in lysis buffer, and the protein was extracted as published previously ${ }^{[22]}$. Briefly, the cells were lysed in buffer containing $50 \mathrm{mmol} / \mathrm{L}$ Tris $\mathrm{pH} 7.4,150 \mathrm{mmol} / \mathrm{L} \mathrm{NaCl}, 1 \%$ Triton $\mathrm{X}-100,10 \%$ glycerol, $5 \mathrm{mmol} / \mathrm{L}$ EDTA, $100 \mu \mathrm{mol} / \mathrm{L}$ sodium vanadate, $1 \mathrm{mmol} / \mathrm{L}$ $\beta$-glycerophosphate, $1 \mathrm{mmol} / \mathrm{L}$ sodium fluoride, $2 \mu \mathrm{g} / \mathrm{mL}$ leupeptin, $10 \mu \mathrm{g} / \mathrm{mL}$ aprotinin, and $1 \mathrm{mmol} / \mathrm{L}$ PMSF. The lysates were collected and centrifuged at $10000 \times \mathrm{g}$ for $10 \mathrm{~min}$ at $4{ }^{\circ} \mathrm{C}$ to pellet the cell debris. The supernatant $(50 \mu \mathrm{g})$ was separated on an SDS-PAGE, and Western blotting was performed as described previously ${ }^{[22]}$. The primary antibodies included monoclonal VEGFR1 (1:500, Santa Cruz Biotechnology), monoclonal VEGFR2 (1:500, Santa Cruz Biotechnology), monoclonal fibronectin (1:5000, BD Biosciences, San Jose, California, USA), monoclonal phospho-MYPT Thr853 (1:1000, Cell Signaling Technology, Danvers, MA, USA), monoclonal phospho-caveolin-1 Y14 (1:1000, BD Biosciences), polyclonal phospho-Src Y416 (1:1000, Cell Signaling Technology) and monoclonal $\beta$-actin (1:5000, Sigma).

\section{Immunoprecipitation}

The kidney cortices were lysed with lysis buffer with 60 mmol/L N-octyl-glucopyranoside as published previously ${ }^{[22]}$. After clarification, equal amounts of lysate were incubated overnight with $2 \mu \mathrm{g}$ of primary antibody rotating at $4{ }^{\circ} \mathrm{C}$ followed by $25 \mu \mathrm{L}$ of protein G-agarose slurry for $1.5 \mathrm{~h}$ at $4^{\circ} \mathrm{C}$. The immunoprecipitates were extensively washed, resuspended in $2 \times$ sample buffer, boiled, and analyzed by immunoblotting.

\section{Quantitative real-time PCR}

Quantitative polymerase chain reaction ( $\mathrm{qPCR}$ ) was performed with RNA extracted from the kidney cortices. Total RNA was isolated using TRIzol reagent according to the manufacturer's instructions (Invitrogen, Carlsbad, California, USA). cDNA was reversely transcribed using the Reverse Transcription kit (Promega, Madison, USA). Quantitative PCR was performed in duplicate using the MiniOpticon (Bio-Rad, Hercules, California, USA). Negative cDNA controls were included for each gene set in all PCR reactions to detect contamination. The thermo-cycle program was set as $10 \mathrm{~min}$ at $94^{\circ} \mathrm{C}$, followed by 30 cycles of at $94^{\circ} \mathrm{C}$ for $30 \mathrm{~s}, 60^{\circ} \mathrm{C}$ for $30 \mathrm{~s}$ and $72^{\circ} \mathrm{C}$ for $1 \mathrm{~min}$.
The expression level for each gene was calculated using the $\Delta \mathrm{Ct}$ method relative to $\beta$-actin.

\section{Statistical analysis}

The data are presented as the mean \pm SEM. Statistical analysis was performed with a one-way ANOVA followed by Tukey's Honestly Significant Difference (HSD) tests for post hoc analysis (SPSS 17.0 for Windows). $P<0.05$ was considered significant. The experiments were repeated multiple times, and the number of repetitions is presented in the figure legends $(n)$.

\section{Results}

Effects of fasudil on metabolic parameters and blood pressure

The metabolic parameters for all animal groups are summarized in Table 1. Diabetic rats had higher plasma glucose concentrations, increased renal hypertrophy (kidney/body weight ratios) and decreased body weights. Administration of fasudil and enalapril did not affect plasma glucose levels, body weights or renal hypertrophy in diabetic rats. Fasudil and enalapril did not affect blood pressure in diabetic rats. Serum creatinine (Table 2) and creatinine clearance (Table 3) were significantly elevated in diabetic rats at month 1 compared to controls. Administration of low-dose fasudil (10 $\mathrm{mg} / \mathrm{kg}$ ) and enalapril had no effect. Middle-dose $(30 \mathrm{mg} / \mathrm{kg})$ and high-dose fasudil (100 mg/kg) improved serum creatinine and creatinine clearance in diabetic rats. No difference in serum creatinine and creatinine clearance was observed at month 3 or 8 between groups. Increased albumin excretion, a hallmark of early diabetic nephropathy, was observed in the diabetic group and was prevented by treatment with middledose or high-dose fasudil (Table 4).

\section{Effects of fasudil on renal morphology and ECM protein}

The glomerular accumulation of PAS-positive matrix was prominent in STZ-induced diabetic rats compared to controls (Figure 1A). Increased glomerular sclerosis was observed in the diabetic group, and sclerosis was prevented in the middledose and high-dose fasudil groups but not in the low-dose fasudil group (Figure 1B). Glomerular basement membrane (GBM) thickness increased in diabetic rats (Figure 1C, 1D). In control animals, the glomerulus podocyte foot processes are arranged regularly and perpendicular to the basement membrane. In the diabetic group, the podocyte foot processes appeared fused together or effaced (arrows) along the surface of GBM (Figure 1D). Treatment with middle- or high-dose fasudil reduced GBM thickening and podocyte foot process effacement.

We assessed renal cortical ROCK activation in each group by Western blotting for phospho-MYPT Thr853. Increased ROCK activation in diabetic renal cortex is prevented by middleand high-dose fasudil treatment. Although there was a trend towards decreased ROCK activation with enalapril, this was not significant (Figure 2A). In addition, fibronectin upregulation in diabetic renal cortex was prevented by middle- and high-dose fasudil treatment, and the effectiveness was similar to enalapril (Figure 2B). These results confirm that renal injury 
Table 1. Clinical characteristics of control rats and STZ-induced diabetic rats untreated or treated with the ROCK inhibitor fasudil at month 8. Data are mean \pm SEM. ${ }^{\mathrm{b}} \mathrm{P}<0.05$ vs control.

\begin{tabular}{lcccccc}
\hline & Control & STZ & STZ+F10 & STZ+F30 & STZ+F100 & STZ+enalapril \\
\hline$n$ & 10 & 11 & 10 & 10 & 9 \\
Glucose (mmol/L) & $3.66 \pm 0.42$ & $29.11 \pm 2.81^{\mathrm{b}}$ & $22.82 \pm 2.31^{\mathrm{b}}$ & $31.11 \pm 3.19^{\mathrm{b}}$ & $33.26 \pm 3.99^{\mathrm{b}}$ & $27.57 \pm 2.32^{\mathrm{b}}$ \\
Weight (g) & $416.5 \pm 17.7$ & $227.9 \pm 22.4^{\mathrm{b}}$ & $225.0 \pm 25.4^{\mathrm{b}}$ & $244.1 \pm 28.1^{\mathrm{b}}$ & $255.7 \pm 18.7^{\mathrm{b}}$ & $236.2 \pm 22.8^{\mathrm{b}}$ \\
Kidney/body weight (mg/g) & $2.50 \pm 0.05$ & $5.74 \pm 0.28^{\mathrm{b}}$ & $4.95 \pm 0.23^{\mathrm{b}}$ & $5.66 \pm 0.25^{\mathrm{b}}$ & $6.03 \pm 0.27^{\mathrm{b}}$ & $4.63 \pm 0.22^{\mathrm{b}}$ \\
Systolic blood pressure (mmHg) & $124.0 \pm 1.2$ & $117.0 \pm 4.2$ & $130.5 \pm 2.5$ & $102 \pm 3.9$ & $112.0 \pm 1.7$ & $112.0 \pm 1.6$ \\
\hline
\end{tabular}

F10, F30, F100: SD rats treated with 10, 30, and $100 \mathrm{mg} / \mathrm{kg}$ fasudil dissolved in drinking water.

Table 2. Serum creatinine level $(\mu \mathrm{mol} / \mathrm{L})$ at month 1,3 , and 8 . Data are mean \pm SEM. ${ }^{\mathrm{b}} P<0.05$ vs control.

\begin{tabular}{lccccc}
\hline Time & Control & STZ & STZ+F10 & STZ+F30 & STZ+F100 \\
\hline Month 1 & $50.8 \pm 6.84$ & $120.9 \pm 42.53^{\mathrm{b}}$ & $77.3 \pm 24.34^{\mathrm{b}}$ & $63.2 \pm 5.29$ & $61.2 \pm 6.67$ \\
Month 3 & $53.3 \pm 5.32$ & $57.0 \pm 11.07$ & $52.7 \pm 3.22$ & $50.1 \pm 7.25$ & $48.5 \pm 7.72$ \\
Month 8 & $46.6 \pm 3.70$ & $40.3 \pm 7.91$ & $41.0 \pm 5.62$ & $37.4 \pm 4.63$ & $37.7 \pm 0.94$ \\
\hline
\end{tabular}

Table 3. The 24-h creatinine clearance $\left(C_{\mathrm{cr}}, \mathrm{mL} \cdot \mathrm{min}^{-1} \cdot 100 \mathrm{~g} \mathrm{BW}^{-1}\right)$ at month 1,3 , and 8. Data are mean $\pm \mathrm{SEM}$. ${ }^{\mathrm{b}} P<0.05 \mathrm{vs}$ control.

\begin{tabular}{lccccc}
\hline Time & Control & STZ & STZ+F10 & STZ+F30 & STZ+F100 \\
\hline Month 1 & $4.43 \pm 0.52$ & $8.29 \pm 1.40^{\mathrm{b}}$ & $8.84 \pm 1.73^{\mathrm{b}}$ & $4.61 \pm 0.55$ & $4.22 \pm 0.47$ \\
Month 3 & $3.56 \pm 0.67$ & $4.83 \pm 0.43$ & $4.54 \pm 0.66$ & $3.96 \pm 0.58$ & $4.35 \pm 0.53$ \\
Month 8 & $4.11 \pm 0.45$ & $3.31 \pm 0.63$ & $3.84 \pm 0.71$ & $3.87 \pm 0.46$ & $4.06 \pm 0.62$ \\
\hline
\end{tabular}

Table 4. Albuminuria (mg/24 h) at month 1,3 , and 8 . Data are mean \pm SEM. ${ }^{b} P<0.05$ vs control.

\begin{tabular}{lcccrr}
\hline Time & Control & STZ & STZ+F10 & STZ+F30 & STZ+F100 \\
\hline Month 1 & $10.09 \pm 0.24$ & $12.43 \pm 0.32$ & $12.19 \pm 0.71$ & $11.32 \pm 0.57$ & $11.55 \pm 0.63$ \\
Month 3 & $10.35 \pm 0.41$ & $26.31 \pm 0.73^{\mathrm{b}}$ & $25.78 \pm 0.64^{\mathrm{b}}$ & $11.38 \pm 0.44$ & $11.29 \pm 0.51$ \\
Month 8 & $10.16 \pm 0.56$ & $28.26 \pm 0.85^{\mathrm{b}}$ & $27.06 \pm 0.93^{\mathrm{b}}$ & $11.62 \pm 0.31$ & $11.06 \pm 0.72$ \\
\hline
\end{tabular}

is characterized by glomerulosclerosis and ECM accumulation in STZ-induced diabetic rats (as expected). ROCK suppression with more than $30 \mathrm{mg} / \mathrm{kg}$ fasudil could ameliorate renal injury in diabetic rats.

Effects of fasudil on VEGF upregulation and its receptors in diabetic kidneys

VEGF and its receptors play a central role in the pathogenesis of diabetic nephropathy. However, whether Rho inhibitors prevent the upregulation of VEGF and VEGF receptors has not been addressed. We first determined the renal cortical expression of VEGF. The VEGF mRNA and protein levels were increased in diabetic rats compared to controls. These levels were restored by middle- and high-dose fasudil (Figure 3A, $3 B)$. The VEGF receptor protein expression was determined in the renal cortex. Immunohistochemistry for VEGFR1 and
VEGFR2 in cortical sections demonstrated strong VEGFR1 and VEGFR2 staining both in the glomeruli and tubular cells in diabetic rats compared to controls (Figure 4A). Middle- and high-dose fasudil prevented VEGFR1 and VEGFR2 upregulation, and enalapril did not affect VEGFR1 and VEGFR2 staining in the renal cortices of diabetic rats. We then determined the VEGFR1 and VEGFR2 protein levels by Western blotting (Figure 4B). Consistent with the immunohistochemistry results, increased VEGFR1 and VEGFR2 protein levels in diabetic renal cortices were inhibited by middle- and high-dose fasudil.

\section{Effects of fasudil on the association of VEGF receptors and caveolin-1 in diabetic kidneys}

Our previous data demonstrated that the association between caveolin-1 and VEGFR2 was involved in the VEGF-induced 

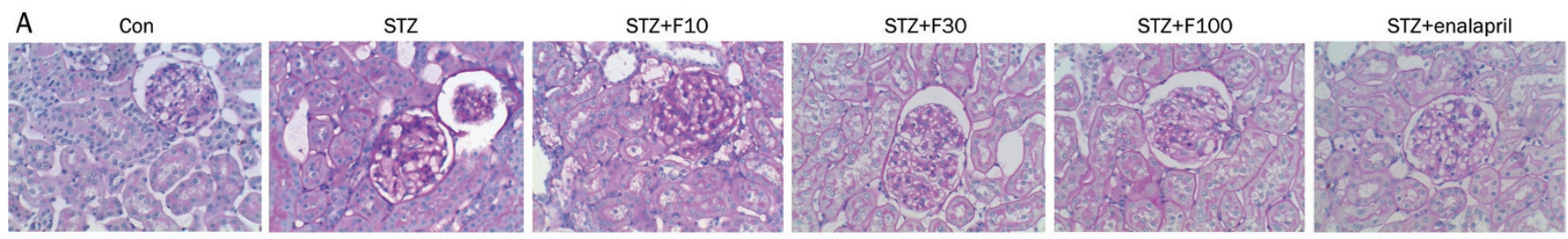

B

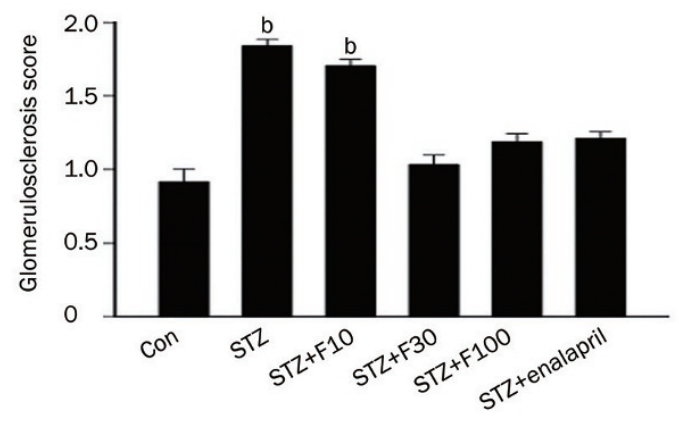

Con

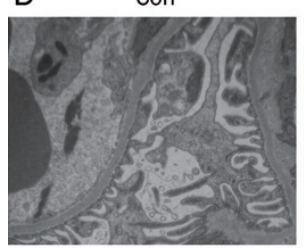

STZ

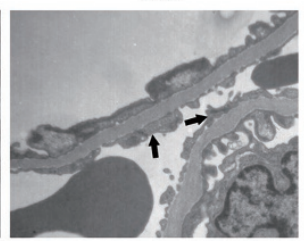

$\mathrm{STZ}+\mathrm{F} 10$

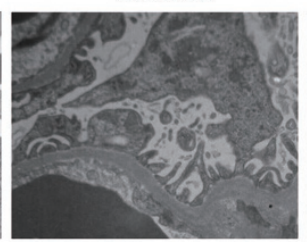

C

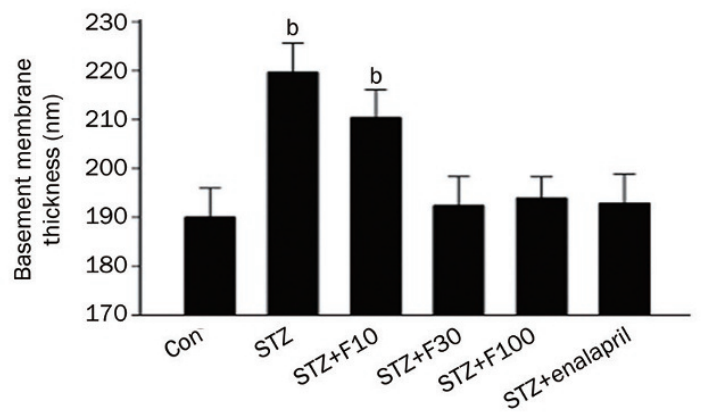

$\mathrm{STZ}+\mathrm{F} 30$

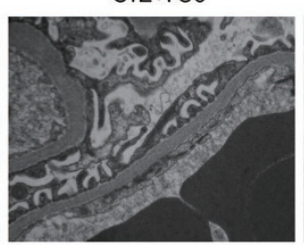

STZ+F100

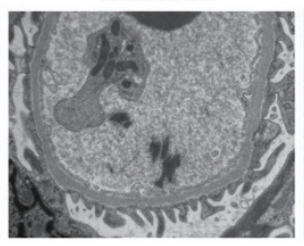

STZ+enalapril

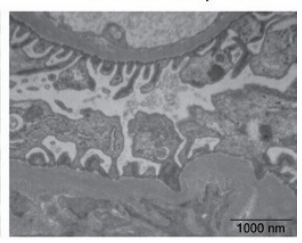

Figure 1. ROCK inhibitor fasudil improves glomerular injury of diabetic nephropathy in vivo. (A) Glomerular histopathology analysis by Periodic Acidschiff (PAS) staining. Representative microphotographs of glomeruli from each group are shown at an original magnification of $400 \times$. (B) Glomerular matrix accumulation was assessed and scores were assigned after blinding to treatment group according to the grading scheme outlined in Materials and methods ( ${ }^{\mathrm{b}} \mathrm{P}<0.05$ vs control). (C) Glomerular basement membrane (GBM) thickness was observed by transmission electron microscopy (TEM), with data summarized in the bar graph ( ${ }^{b} P<0.05$ vs control). (D) Representative microphotographs of GBM are shown at magnification $10000 \times$. Black arrows denote focal areas of foot process effacement. F10, F30, and F100: SD rats treated with 10, 30, and $100 \mathrm{mg} / \mathrm{kg}$ fasudil daily.

fibronectin upregulation within mesangial cells. This association was also increased in the renal cortices of diabetic rats, although both VEGFR1 and VEGFR2 receptor protein levels were increased in diabetic rats ${ }^{[23]}$. Here, we assessed the effects of fasudil on the association between VEGFR2 and caveolin-1 by immunoprecipitation of caveolin-1 from the renal cortices. The increased association between VEGFR2 and caveolin-1 in diabetic rats was prevented by middle- and high-dose fasudil but not by enalapril (Figure 5). These data indicate that the VEGFR2/caveolin-1 signaling pathway is likely blocked by ROCK inhibition during diabetic nephropathy.

\section{Effects of fasudil on the phosphorylation of Src and caveolin-1 in diabetic glomeruli}

Src-family kinases are the only known caveolin-1 Y14 kinases $^{[24,25]}$, and our previous studies demonstrated that TGF$\beta$ - and VEGF-induced Src activation mediated caveolin-1 phosphorylation at Y14 followed by fibronectin synthesis in mesangial cells ${ }^{[23,26]}$. Because fasudil prevented VEGF upregulation in diabetic kidneys, we investigated the effect of fasudil on Src and caveolin-1 phosphorylation levels. We examined the auto-phosphorylation of Y416 on Src, which is indicative of increased Src activity, and caveolin-1 phosphorylation on Y14 in glomeruli by immunoblotting. Phosphorylated Src and caveolin-1 were significantly increased in glomerular isolates compared to controls. Treatment with middle- and high-dose fasudil partially blocked Src and caveolin-1 phosphorylation; enalapril had no effect (Figure 6A, 6B). These results suggest that the beneficial effect of fasudil is probably mediated through reversing the enhanced Src kinase activity and downstream caveolin-1 phosphorylation in diabetic rats.

\section{Discussion}

Diabetic nephropathy is the leading cause of end-stage renal disease, which is characterized by GBM thickening, mesangial expansion and glomerular hypertrophy in its early stages followed by ECM accumulation, glomerulosclerosis, and tubulointerstitial fibrosis. Recent studies have demonstrated that treatment with fasudil, a ROCK inhibitor, prevents the development of experimental diabetic nephropathy by inhibiting the signaling pathways involving angiotensin II, TGF- $\beta$, and ECM accumulation ${ }^{[9,10,27]}$. However, whether fasudil blocks VEGF signaling has not been addressed yet. We identified a previously unrecognized renoprotective mechanism for the 
A
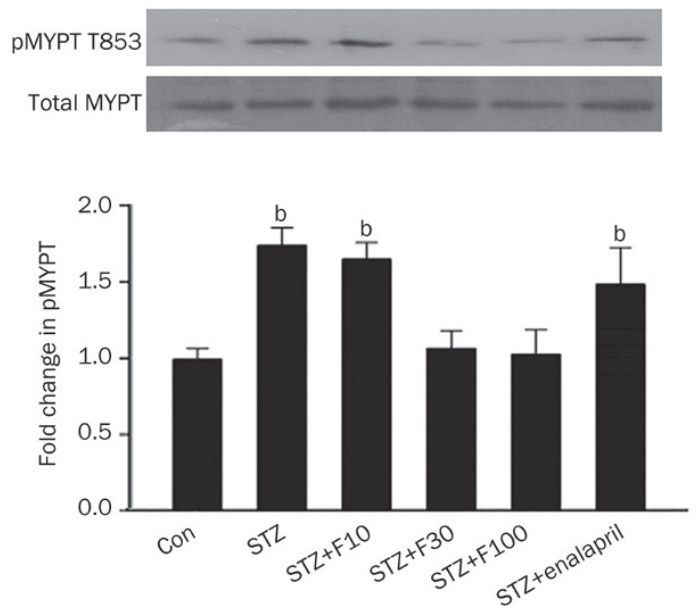

B
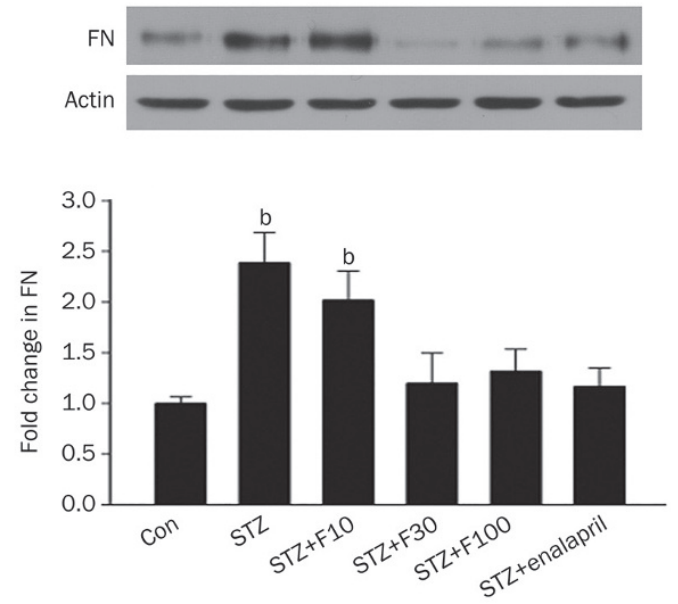

Figure 2. ROCK inhibitor fasudil prevents MYPT phosphorylation and fibronectin upregulation in diabetic kidneys. (A) MYPT phosphorylation (Thr 853) was determined by Western blotting as an indicator of renal cortical ROCK activity $\left({ }^{\mathrm{b}} \mathrm{P}<0.05\right.$ vs control). (B) The protein level of fibronectin (FN) was analyzed by Western blotting in renal cortical homogenates, with actin used as loading control $\left({ }^{b} P<0.05\right.$ vs control). Representative blots are shown in the upper panel for both $\mathrm{A}$ and $\mathrm{B}$, with the graph below summarizing data from all animals.

effects of fasudil by preventing the upregulation of VEGF and its receptors, VEGFR2/caveolin-1 association and enhanced fibronectin synthesis during STZ-induced diabetic nephropathy.

The diabetic rats developed kidney hypertrophy, mild albuminuria, and progressive glomerulosclerosis. Chronic treatment with middle-dose $(30 \mathrm{mg} / \mathrm{kg})$ and high-dose $(100$ $\mathrm{mg} / \mathrm{kg}$ ) fasudil ameliorated the kidney injuries with no effect on blood pressure and glucose, which is consistent with previous studies ${ }^{[10,12]}$. Although there was a trend towards decreased blood pressure in the middle-dose fasudil group, this trend was not significant. Komers et al reported that acute administration of fasudil decreased blood pressure in both control and diabetic rats ${ }^{[28]}$. However, hypertension is the most common adverse event associated with VEGFR-tyrosine

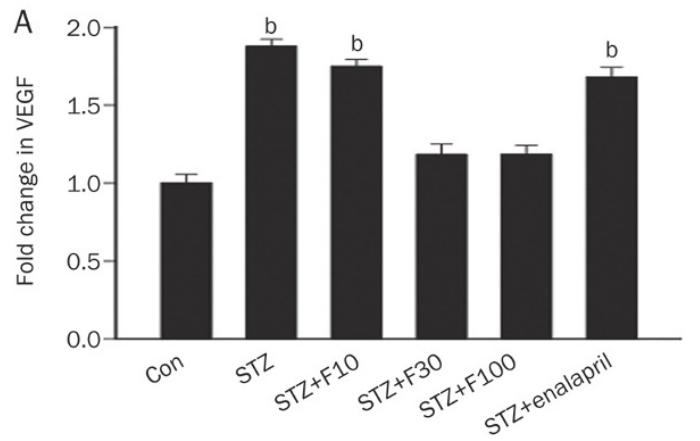

B
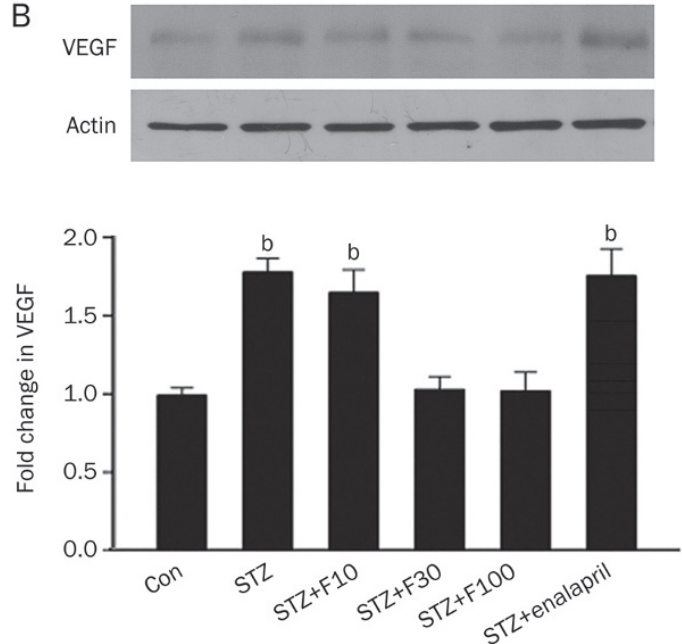

Figure 3. Gene and protein expression of VEGF is reduced by ROCK inhibitor fasudil in diabetic kidneys. (A) VEGF gene expression was detected in renal cortical samples by real-time PCR. (B) VEGF protein level in renal cortex was assessed by Western blotting, with actin used as loading control. Data are shown as fold induction compared with control $\left({ }^{\mathrm{b} P}<0.05\right.$ vs control).

kinase inhibitors, and ROCK inhibition blunts sunitinib(VEGF antagonist) -induced hypertension ${ }^{[29]}$. Further studies are required to determine whether fasudil has specific role in the control of blood pressure. Low-dose $(10 \mathrm{mg} / \mathrm{kg})$ fasudil had no effect in this study, which is different from previous studies $^{[4,27]}$, and may be related to the differences in animal models, treatment periods and protocols.

In the present study, we evaluated the effects of fasudil on serum creatinine and creatinine clearance throughout the course of the disease and extended previous results that have not been explored before. At month 1, diabetic rats demonstrated increased serum creatinine and creatinine clearance, which was improved by middle- and high-dose fasudil but not by enalapril. Our data indicated that fasudil may improve the enhanced GFR during the very early stage of nephropathy. No difference in serum creatinine or creatinine clearance was observed between groups at month 3 or 8 , which agrees with previous studies ${ }^{[5,27]}$

VEGF and its receptors are widely expressed in different cell types in the kidney ${ }^{[30,31]}$. VEGF plays a central role in the 
A
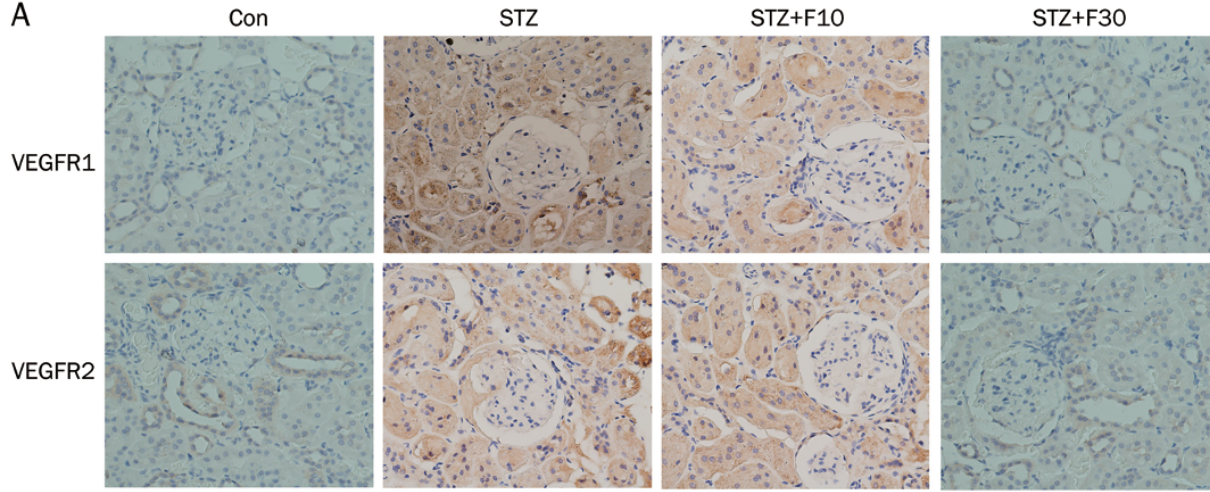

STZ+F100
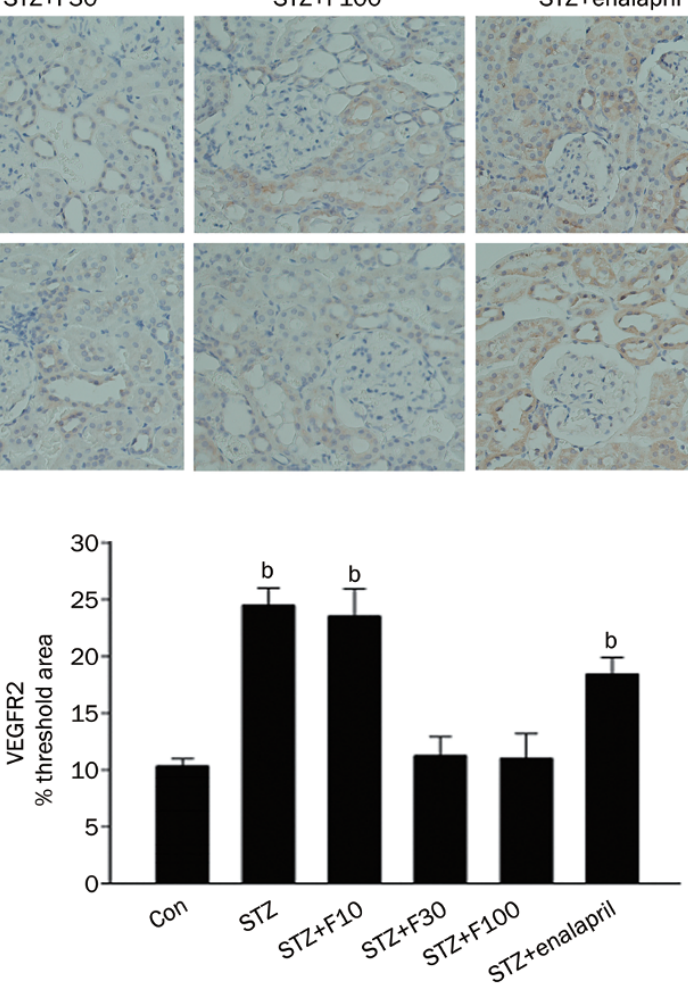

B
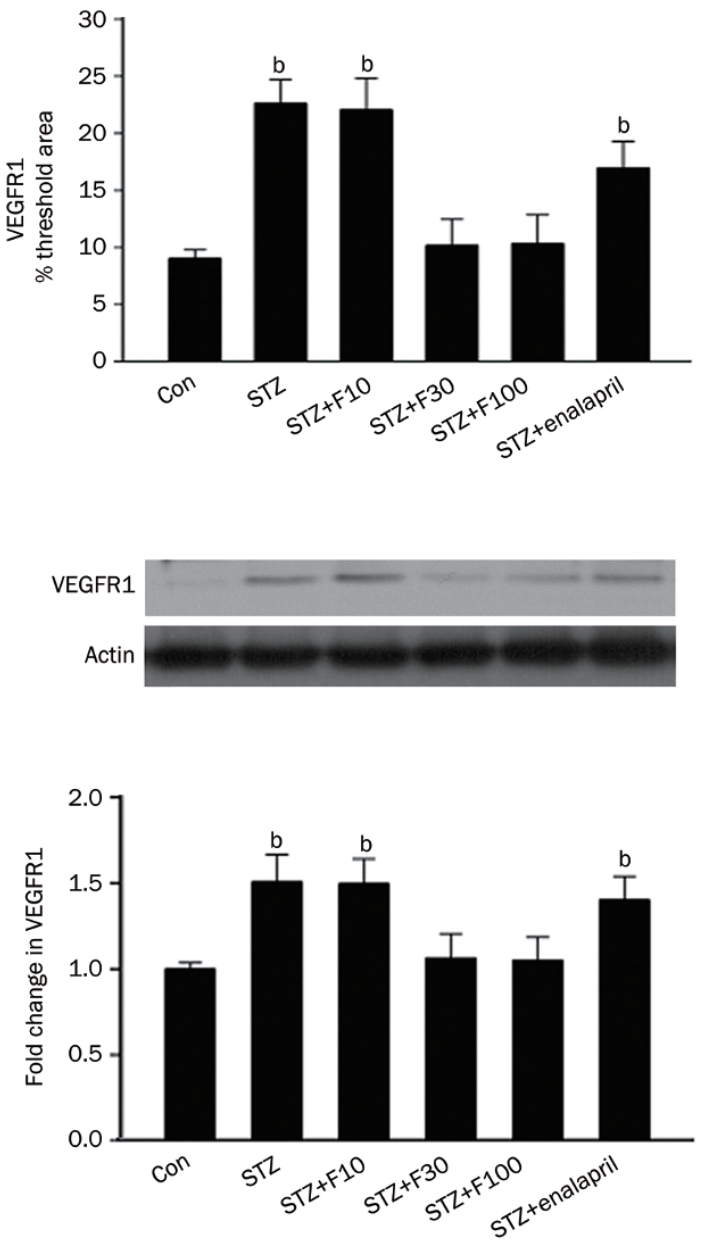
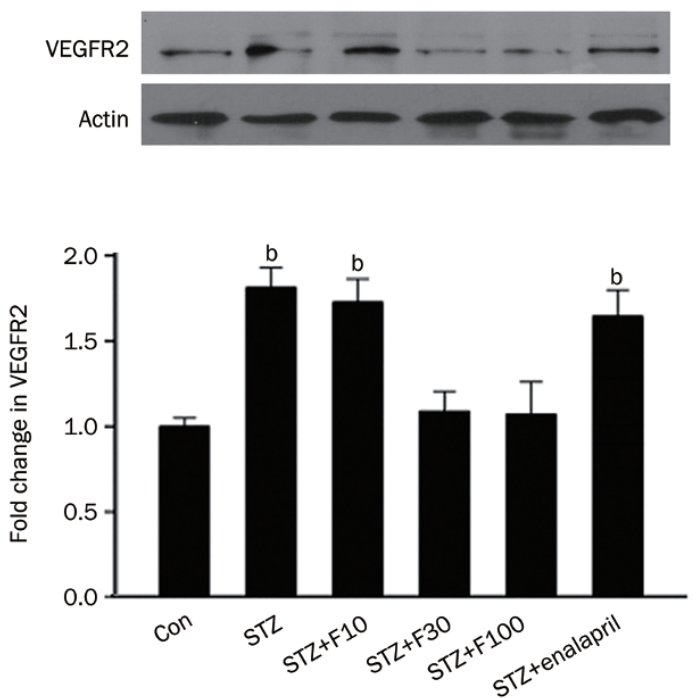

Figure 4. ROCK inhibitor fasudil prevents upregulation of VEGFR1 and VEGFR2 in diabetic kidneys. (A) Representative microphotographs of VEGFR1 and VEGFR2 immunostaining $(400 \times)$ are shown. Computer-aided analysis of renal cortical VEGFR1 and VEGFR2 expressed as percent area $\left({ }^{\mathrm{b}} P<0.05\right.$ vs control). (B) VEGFR1 and VEGFR2 protein levels in renal cortex were assessed by Western blotting, with actin used as loading control $\left({ }^{\mathrm{b}} P<0.05 \mathrm{vs}\right.$ control).

pathogenesis of diabetic nephropathy through its modulatory effects on glomerular hypertrophy, proteinuria and ECM production $^{[32-34]}$. However, little is known regarding the effects of fasudil on VEGF regulation. The present study demonstrated that fasudil inhibited VEGF upregulation in diabetic rat renal cortex. Similarly Peng et al ${ }^{[10]}$ demonstrated that the Rho inhibitor Y-27632 prevented high glucose-induced upregulation of VEGF transcription in mesangial cells. These results suggest that VEGF upregulation is probably dependent on RhoA/ROCK signaling. Given these observations, we also assessed the effects of fasudil on the protein levels of two VEGF receptors, VEGFR1 and VEGFR2, by immunohis- 

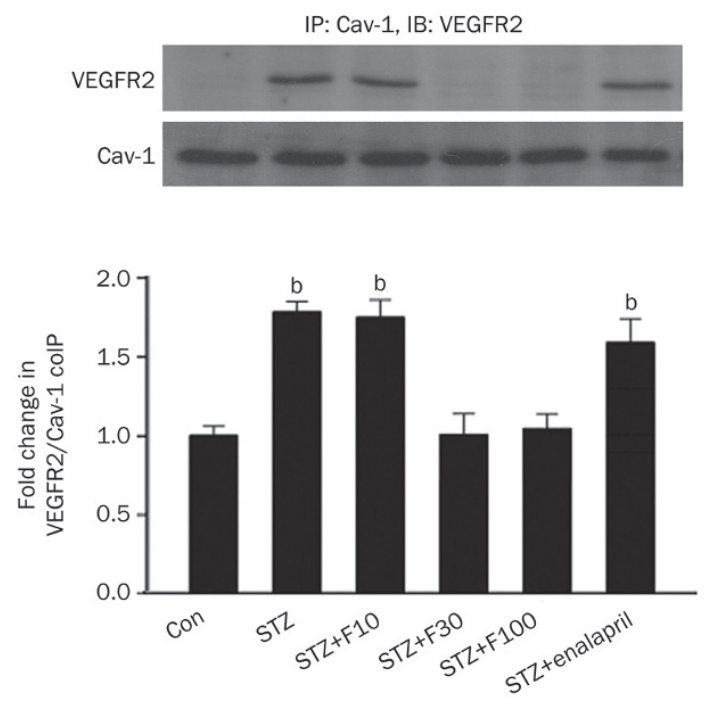

Figure 5. The association between VEGFR2 with caveolin-1 is inhibited by fasudil in diabetic kidneys. Caveolin-1 was immunopricipitated from renal cortical homogenates and its association with VEGFR2 was assessed by Western blotting. ${ }^{b} P<0.05$ vs control..

tochemistry and Western blotting in diabetic rat renal cortex. Increased VEGFR1 and VEGFR2 protein levels in the diabetic group were inhibited by middle- and high-dose fasudil supporting a potential role of fasudil in preventing VEGF signaling.

Although VEGF signaling is activated during diabetic nephropathy, little is known regarding the function of different VEGF receptors. $\mathrm{VEGF}_{165}$, the most biologically active VEGF isoform, predominantly signals through VEGFR2, although $\mathrm{VEGF}_{165}$ can also be "retained" by VEGFR1, which has limited signaling potential ${ }^{[35]}$. VEGFR2 has been localized in caveolae in several cell lines, and caveolae have been implicated in VEGF-induced ERK2/1 signaling and permeability in vascular endothelial cells ${ }^{[36,37]}$. Caveolae are specialized plasma membrane microdomains morphologically defined as 50- to 100-nm omega-shaped invaginations according to electron microscopy observations ${ }^{[38,39]}$. Caveolin- 1 functions as a scaffolding protein by directly interacting with various signaling molecules and integrates specific transmembrane signaling pathways in the caveolae, including VEGF signaling ${ }^{[40,41]}$. Our previous studies demonstrated that the VEGFR2/caveolin-1 association plays an important role in ECM accumulation in mesangial cells ${ }^{[23]}$. Recent studies have demonstrated that selective stimulation of VEGFR2 accelerates progressive renal disease $^{[42]}$. Thus, we investigated the effects of fasudil on the association between VEGFR2 and caveolin-1 within the diabetic renal cortex. Increased association between VEGFR2 and caveolin-1 in the diabetic groups was prevented by middleand high-dose fasudil indicating that the VEGFR2/caveolin-1 signaling pathway is probably prevented by ROCK inhibition in diabetic nephropathy.

We have previously demonstrated that the VEGFR2/caveo-
A
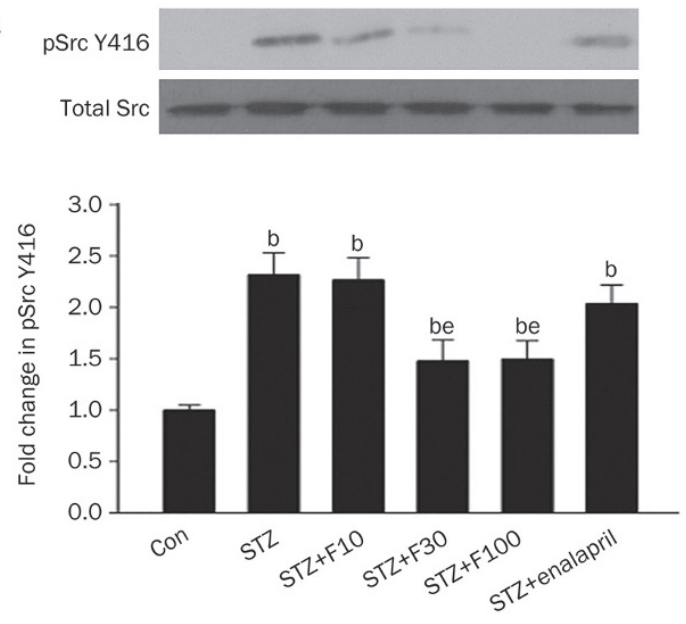

B
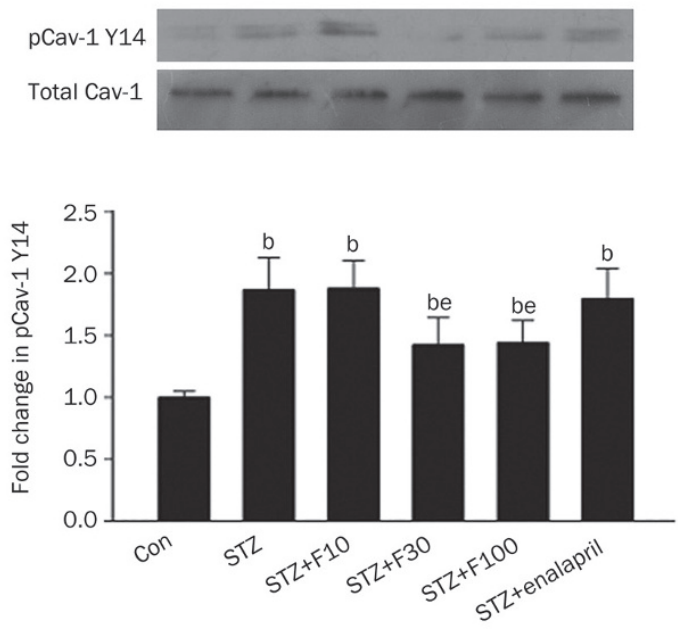

Figure 6. Src activation and caveolin-1 phosphorylation on Y14 in diabetic glomeruli is partly blocked by ROCK inhibitor fasudil. (A) Y416phosphorylated Src (pSrc Y416) in glomerular isolates was assessed by Western blotting. (B) Y14-phosphorylated caveolin-1 (pCav-1 Y14) in glomerular isolates was assessed by Western blotting. ${ }^{\mathrm{b}} P<0.05 \mathrm{vs}$ control. ${ }^{e} P<0.05$ vs diabetic group.

lin- 1 association is mediated by the caveolin- 1 /Src association, Src activation and further modification of caveolin-1 phosphorylation on tyrosine 14 via Src kinase during VEGFinduced matrix upregulation in mesangial cells ${ }^{[23]}$. Src activation also occurs in the kidneys of mice with STZ-induced type 1 diabetes. The Src inhibitor PP2 prevents albuminuria, glomerular matrix protein accumulation, GBM thickening, and podocyte depletion ${ }^{[43]}$. Therefore, this study investigated the effects of fasudil on Src/caveolin-1 signaling. Middle- and high-dose fasudil partially inhibited Src activation and caveolin-1 phosphorylation at Y14 suggesting that the renoprotective effect of fasudil is at least in partially mediated through blocking Src kinase activation and caveolin-1 phosphorylation in diabetic rats. Although the ACE inhibitor enalapril is effective in the prevention of early diabetic nephropathy ${ }^{[4]}$, it cannot block VEGFR2/Src/caveolin-1 signaling. 
In conclusion, the results of the present study provide evidence regarding the renoprotective mechanisms of the ROCK inhibitor fasudil concerning VEGF/Src/caveolin-1 signaling, which could be a potential therapeutic target for the treatment of diabetic nephropathy.

\section{Acknowledgements}

This work was supported by grants from the National Natural Science Foundation of China (81070573 and 81370819).

\section{Author contribution}

Jing JIN and Chao PENG performed the experiments; Su-zhen WU and Hong-min CHEN collected and analyzed the data; Bai-fang ZHANG designed the experiments and prepared the manuscript.

\section{References}

1 Bach LA. Rho kinase inhibition: a new approach for treating diabetic nephropathy? Diabetes 2008; 57: 532-3.

2 Komers R. Rho kinase inhibition in diabetic nephropathy. Curr Opin Nephrol Hypertens 2011; 20: 77-83.

3 Hagiwara S, Kantharidis P, Cooper ME. What are new avenues for renal protection, in addition to RAAS inhibition? Curr Hypertens Rep 2012; 14: 100-10.

4 Gojo A, Utsunomiya K, Taniguchi K, Yokota T, Ishizawa S, Kanazawa $Y$, et al. The Rho-kinase inhibitor, fasudil, attenuates diabetic nephropathy in streptozotocin-induced diabetic rats. Eur J Pharmacol 2007; 568: 242-7.

5 Kikuchi Y, Yamada M, Imakiire T, Kushiyama T, Higashi K, Hyodo N, et al. A Rho-kinase inhibitor, fasudil, prevents development of diabetes and nephropathy in insulin-resistant diabetic rats. J Endocrinol 2007; 192: 595-603.

6 Etienne-Manneville S, Hall A. Rho GTPases in cell biology. Nature 2002; 420: 629-35.

7 Loirand G, Guerin P, Pacaud P. Rho kinases in cardiovascular physiology and pathophysiology. Circ Res 2006; 98: 322-34.

8 Ridley AJ. Rho family proteins: coordinating cell responses. Trends Cell Biol 2001; 11: 471-7.

9 Hayashi K, Wakino S, Kanda T, Homma K, Sugano N, Saruta T. Molecular mechanisms and therapeutic strategies of chronic renal injury: role of rho-kinase in the development of renal injury. J Pharmacol Sci 2006; 100: 29-33.

10 Peng F, Wu D, Gao B, Ingram AJ, Zhang B, Chorneyko K, et al. RhoA/ Rho-kinase contribute to the pathogenesis of diabetic renal disease. Diabetes 2008; 57: 1683-92.

11 Arita R, Hata Y, Nakao S, Kita T, Miura M, Kawahara S, et al. Rho kinase inhibition by fasudil ameliorates diabetes-induced microvascular damage. Diabetes 2009; 58: 215-26.

12 Komers R, Oyama TT, Beard DR, Tikellis C, Xu B, Lotspeich DF, et al. Rho kinase inhibition protects kidneys from diabetic nephropathy without reducing blood pressure. Kidney Int 2011; 79: 432-42.

13 Matoba K, Kawanami D, Okada R, Tsukamoto M, Kinoshita J, Ito $\mathrm{T}$, et al. Rho-kinase inhibition prevents the progression of diabetic nephropathy by downregulating hypoxia-inducible factor $1 \alpha$. Kidney Int 2013; 84: 545-54.

14 Davies SP, Reddy H, Caivano M, Cohen P. Specificity and mechanism of action of some commonly used protein kinase inhibitors. Biochem J 2000; 351: 95-105.

15 Advani A. Vascular endothelial growth factor and the kidney: some- thing of the marvellous. Curr Opin Nephrol Hypertens 2014; 23: 87-92.

16 Liu E, Morimoto M, Kitajima S, Koike T, Yu Y, Shiiki H, et al. Increased expression of vascular endothelial growth factor in kidney leads to progressive impairment of glomerular functions. J Am Soc Nephrol 2007; 18: 2094-104.

17 Chen S, Ziyadeh FN. Vascular endothelial growth factor and diabetic nephropathy. Curr Diab Rep 2008; 8: 470-6.

18 Mironidou-Tzouveleki M, Tsartsalis S, Tomos C. Vascular endothelial growth factor (VEGF) in the pathogenesis of diabetic nephropathy of type 1 diabetes mellitus. Curr Drug Targets 2011; 12: 107-14.

19 Cross MJ, Dixelius J, Matsumoto T, Claesson-Welsh L. VEGF-receptor signal transduction. Trends Biochem Sci 2003; 28: 488-94.

20 Shibuya M, Claesson-Welsh L. Signal transduction by VEGF receptors in regulation of angiogenesis and lymphangiogenesis. Exp Cell Res 2006; 312: 549-60.

21 Raij L, Azar S, Keane W. Mesangial immune injury, hypertension, and progressive glomerular damage in Dahl rats. Kidney Int 1984; 26 : 137-43.

22 Krepinsky JC, Ingram AJ, Tang D, Wu D, Liu L, Scholey JW. Nitric oxide inhibits stretch-induced MAPK activation in mesangial cells through RhoA inactivation. J Am Soc Nephrol 2003; 14: 2790-800.

23 Wu TT, Zhang BF, Ye F, Xiao ZL. A potential role for caveolin-1 in VEGFinduced fibronectin upregulation in mesangial cells: involvement of VEGFR2 and Src. Am J Physiol Renal Physiol 2013; 304: F820-30.

$24 \mathrm{Cao} \mathrm{H}$, Courchesne WE, Mastick CC. A phosphotyrosine-dependent protein interaction screen reveals a role for phosphorylation of caveolin-1 on tyrosine 14: recruitment of C-terminal Src kinase. J Biol Chem 2002; 277: 8771-4.

25 Li S, Seitz R, Lisanti MP. Phosphorylation of caveolin by src tyrosine kinases. The alpha-isoform of caveolin is selectively phosphorylated by v-Src in vivo. J Biol Chem 1996; 271: 3863-8.

26 Peng F, Zhang BF, Wu D, Ingram AJ, Gao B, Krepinsky JC. TGF $\beta$ induced RhoA activation and fibronectin production in mesangial cells require caveolae. Am J Physiol Renal Physiol 2008; 295: F153-64.

27 Kolavennu V, Zeng L, Peng H, Wang Y, Danesh FR. Targeting of RhoA/ ROCK signaling ameliorates progression of diabetic nephropathy independent of glucose control. Diabetes 2008; 57: 714-23.

28 Komers R, Oyama TT, Beard DR, Anderson S. Effects of systemic inhibition of Rho kinase on blood pressure and renal hemodynamics in diabetic rats. Br J Pharmacol 2011; 162: 163-74.

29 Grisk O, Koenen A, Meissner T, Donner A, Braun D, Steinbach A, et al. Rho kinase inhibition mitigates sunitinib-induced rise in arterial pressure and renal vascular resistance but not increased renal sodium reabsorption. J Hypertens 2014; 32: 2199-210.

30 Robert B, Zhao X, Abrahamson DR. Coexpression of neuropilin-1, FIk1, and VEGF(164) in developing and mature mouse kidney glomeruli. Am J Physiol Renal Physiol 2000; 279: F275-82.

31 Thomas S, Vanuystel J, Gruden G, Rodriguez V, Burt D, Gnudi L, et al. Vascular endothelial growth factor receptors in human mesangium in vitro and in glomerular disease. J Am Soc Nephrol 2000; 11: 123643.

32 Kang DH, Johnson RJ. Vascular endothelial growth factor: a new player in the pathogenesis of renal fibrosis. Curr Opin Nephrol Hypertens 2003; 12: 43-9.

33 Nakagawa T. A new mouse model resembling human diabetic nephropathy: uncoupling of VEGF with eNOS as a novel pathogenic mechanism. Clin Nephrol 2009; 71: 103-9.

34 Sato W, Kosugi T, Zhang L, Roncal CA, Heinig M, Campbell-Thompson $M$, et al. The pivotal role of VEGF on glomerular macrophage infiltration in advanced diabetic nephropathy. Lab Invest 2008; 88: 
949-61.

35 Park JE, Chen HH, Winer J, Houck KA, Ferrara N. Placenta growth factor. Potentiation of vascular endothelial growth factor bioactivity, in vitro and in vivo, and high affinity binding to Flt-1 but not to Flk-1/ KDR. J Biol Chem 1994; 269: 25646-54.

36 Feng Y, Venema VJ, Venema RC, Tsai N, Behzadian MA, Caldwell RB. VEGF-induced permeability increase is mediated by caveolae. Invest Ophthalmol Vis Sci 1999; 40: 158-67.

37 Labrecque L, Royal I, Surprenant DS, Patterson C, Gingras D, Béliveau R. Regulation of vascular endothelial growth factor receptor-2 activity by caveolin-1 and plasma membrane cholesterol. Mol Biol Cell 2003; 14: 334-47.

38 Anderson RG. The caveolae membrane system. Annu Rev Biochem 1998; 67: 199-225.

39 Parat MO. The biology of caveolae: achievements and perspectives. Int Rev Cell Mol Biol 2009; 273: 117-62.

40 Liao WX, Feng L, Zhang H, Zheng J, Moore TR, Chen DB. Compartmentalizing VEGF-induced ERK2/1 signaling in placental artery endothelial cell caveolae: a paradoxical role of caveolin-1 in placental angiogenesis in vitro. Mol Endocrinol 2009; 23: 1428-44.

41 Liu J, Razani B, Tang S, Terman BI, Ware JA, Lisanti MP. Angiogenesis activators and inhibitors differentially regulate caveolin-1 expression and caveolae formation in vascular endothelial cells. Angiogenesis inhibitors block vascular endothelial growth factor-induced downregulation of caveolin-1. J Biol Chem 1999; 274: 15781-6.

42 Sato W, Tanabe K, Kosugi T, Hudkins K, Lanaspa MA, Zhang L, et al. Selective stimulation of VEGFR2 accelerates progressive renal disease. Am J Pathol 2011; 179: 155-66.

43 Taniguchi K, Xia L, Goldberg HJ, Lee KW, Shah A, Stavar L, et al. Inhibition of Src kinase blocks high glucose-induced EGFR transactivation and collagen synthesis in mesangial cells and prevents diabetic nephropathy in mice. Diabetes 2013; 62: 3874-86.

44 Lewis EJ, Hunsicker LG, Bain RP, Rohde RD. The effect of angiotensinconverting-enzyme inhibition on diabetic nephropathy. The Collaborative Study Group. N Engl J Med 1993; 329: 1456-62. 